Canadian Journal of Applied Linguistics

\title{
Les représentations associées aux français nationaux, aux espaces géographiques et aux locuteurs dans les manuels de français langue étrangère et de français langue seconde : étude comparée entre la France et le Québec
}

\section{Marie Duchemin}

Volume 20, numéro 2, 2017

Special Issue: Current Perspectives on Oral Communicative Competence of French Second Language Speakers

URI : https://id.erudit.org/iderudit/1042676ar

DOI : https://doi.org/10.7202/1042676ar

Aller au sommaire du numéro

Éditeur(s)

University of New Brunswick

ISSN

1920-1818 (numérique)

Découvrir la revue

\section{Citer cet article}

Duchemin, M. (2017). Les représentations associées aux français nationaux, aux espaces géographiques et aux locuteurs dans les manuels de français langue étrangère et de français langue seconde : étude comparée entre la France et le Québec. Canadian Journal of Applied Linguistics / Revue canadienne de linguistique appliquée, 20(2), 51-70. https://doi.org/10.7202/1042676ar
Résumé de l'article

Avec l'importance grandissante accordée au développement de la compétence de communication et tout particulièrement à la compétence sociolinguistique en classe de langues, les manuels de français langue seconde ou étrangère s'intéressent désormais à la pluralité de la langue, notamment en présentant différents variétés et usages sociolinguistiques qui se font entendre, adaptant ainsi le contenu aux échanges dans l'espace francophone. Toutefois, le traitement des français nationaux et de leurs locuteurs entre souvent en conflit avec l'enseignement d'une langue de scolarisation se voulant uniforme. Cette étude, qui s'inspirait de la linguistique de discours comparative, proposait d'examiner, par l'intermédiaire d'un corpus de 10 manuels destinés à un public adolescent et adulte, les représentations associées aux français nationaux, aux territoires géographiques et aux locuteurs dans des manuels français et québécois. Les résultats ont démontré que les représentations associées aux français nationaux variaient grandement en fonction du lieu d'édition des manuels. Les manuels québécois présentaient une seule variété, dont les traits informels étaient mis en évidence tandis que les manuels français, présentant plusieurs français nationaux, utilisaient la variété française comme point de référence pour la comparaison.
Copyright (c) Marie Duchemin, 2017

Attribution 3.0 Unported (CC BY 3.0)
Ce document est protégé par la loi sur le droit d'auteur. L’utilisation des services d'Érudit (y compris la reproduction) est assujettie à sa politique d'utilisation que vous pouvez consulter en ligne.

https://apropos.erudit.org/fr/usagers/politique-dutilisation/ 


\title{
Les représentations associées aux français nationaux, aux espaces géographiques et aux locuteurs dans les manuels de français langue étrangère et de français langue seconde : étude comparée entre la France et le Québec
}

\author{
Marie Duchemin \\ Université Laval
}

\begin{abstract}
Résumé
Avec l'importance grandissante accordée au développement de la compétence de communication et tout particulièrement à la compétence sociolinguistique en classe de langues, les manuels de français langue seconde ou étrangère s'intéressent désormais à la pluralité de la langue, notamment en présentant différents variétés et usages sociolinguistiques qui se font entendre, adaptant ainsi le contenu aux échanges dans l'espace francophone. Toutefois, le traitement des français nationaux et de leurs locuteurs entre souvent en conflit avec l'enseignement d'une langue de scolarisation se voulant uniforme. Cette étude, qui s'inspirait de la linguistique de discours comparative, proposait d'examiner, par l'intermédiaire d'un corpus de 10 manuels destinés à un public adolescent et adulte, les représentations associées aux français nationaux, aux territoires géographiques et aux locuteurs dans des manuels français et québécois. Les résultats ont démontré que les représentations associées aux français nationaux variaient grandement en fonction du lieu d'édition des manuels. Les manuels québécois présentaient une seule variété, dont les traits informels étaient mis en évidence tandis que les manuels français, présentant plusieurs français nationaux, utilisaient la variété française comme point de référence pour la comparaison.
\end{abstract}

\begin{abstract}
With the growing importance given to the development of communicative competence and especially to sociolinguistic competence in language classes, French as a second or foreign language textbooks are now focusing on the plurality of language use, in particular by presenting different varieties and sociolinguistic uses, thus adapting the content to exchanges in the French-speaking world. However, the treatment of French national varieties and their speakers often conflicts with the teaching of a language of instruction, which is intended to be uniform. This study, which was based on a comparative discourse approach, proposed to examine, through a corpus of 10 textbooks intended for adolescent and adult audiences, representations associated with national French, geographic territories and speakers in French and Quebec textbooks. The results showed that the representations associated with national French varied greatly according to the place where the textbooks were published. Quebec textbooks had only one variety and the informal traits were highlighted, while the French textbooks, presenting several French varieties, used the variety from France as a point of reference for comparison.
\end{abstract}




\section{Les représentations associées aux français nationaux, aux espaces géographiques et aux locuteurs dans les manuels de français langue étrangère et de français langue seconde : étude comparée entre la France et le Québec}

\section{Introduction}

Le français, comme toute langue, ne représente pas une entité homogène, surtout lorsque l'on s'attarde aux usages et aux pratiques de ses nombreux locuteurs. Cette pluralité des formes est propre au fait que la langue varie en fonction de différents facteurs, tels que le contexte géographique et social, les locuteurs ainsi que les relations que ces derniers entretiennent entre eux. La connaissance des normes et des usages qui appartiennent à un groupe de locuteurs est, comme l'avançait Hymes (1972), une partie essentielle de la compétence de communication de tout locuteur. Bachman (1990) a fait référence à cet aspect de la compétence de communication sous le terme de compétence sociolinguistique, qu'il a défini comme suit : "sensitivity to the conventions of language use . . determined by the specific language use context, [which] enables us to perform language functions in ways that are appropriate to that [specific] context $»$ (p. 94). Dans le modèle proposé par Bachman (1990, 2010), la maitrise de cette compétence intègre la connaissance des différents registres de langue, l'habileté à interpréter les références culturelles, la spontanéité du discours ainsi que la perception des différences dans les dialectes et variétés d'une langue. Pour développer sa compétence sociolinguistique, il faut prendre part à des expériences langagières riches et diversifiées (voir notamment Chevrot, Chabanel et Dugua, 2007 ; Nardy, Chevrot et Barbu, 2013) qui prennent en compte les pratiques linguistiques de nombreux groupes de locuteurs d'une même langue. Pour les apprenants de langues étrangères, cette exposition se fait initialement grâce au matériel pédagogique utilisé en salle de classe (Monerris Oliveras, 2015).

Plusieurs manuels français et québécois récents mettent de l'avant une vision plurielle du français, en intégrant notamment des traits propres à la langue courante parlée

(p. ex. À mots découverts, Transmission 4, Transmission 5). Il est donc pertinent d'observer et d'analyser comment ce rapport à la pluralité est exploité, et ce, par l'étude des représentations culturelles et linguistiques véhiculées à l'intérieur des manuels. La contribution principale de cet article est d'ouvrir le dialogue sur la langue afin de brosser un portrait réel des représentations associées à des variétés nationales, ${ }^{1}$ aux espaces géographiques et aux locuteurs dans les manuels de français. Pour ce faire, nous avons eu recours à la linguistique de discours comparative pour l'analyse du corpus de 10 manuels.

\section{Revue des écrits}

L'étude de manuels dans le domaine des représentations s'avère un outil incontournable puisque, comme l'ont avancé Chiss et Cicurel (2005) : « Les manuels qui constituent et réfractent tout à la fois les cultures éducatives permettent un accès aux variables anthropologiques dont on ne soupçonne pas toujours l'amplitude » (p. 7). C'est pourquoi les manuels ont fait l'objet d'études sur les représentations, et ce, tant en enseignement de la langue maternelle (L1) qu'en enseignement d'une langue seconde (L2).

Les études s'intéressant aux représentations dans les manuels en contexte de français L1 ont abordé de nombreux sujets, dont les représentations des enfants et des adolescents ainsi que de l'apprentissage (von Münchow, 2004a, 2009). von Münchow 
(2004a) a proposé une analyse comparative de manuels d'Allemagne et de France. Les résultats ont démontré que la culture didactique, anthropologique et linguistique dans laquelle les manuels s'inscrivaient était à l'origine des différences de représentations du lecteur, et donc, du traitement de la langue et de son apprentissage. D'autres chercheurs se sont plutôt intéressés à ce médium en L2 et ont abordé la question des représentations interculturelles. N. Auger (2003) a démontré que les représentations de soi étaient toujours plus valorisées que celles de l'Autre dans des manuels de français langue étrangère (FLE) et qu'elles étaient grandement influencées par les contextes historiques, politiques et sociaux inhérents au lieu d'édition des manuels. Dans le même ordre d'idée, Chapelle (2014) a étudié les représentations du Québec et du Canada dans les manuels américains de français langue seconde (FLS). L'analyse chronologique des manuels a mis de l'avant l'augmentation des contenus relatifs à ces deux espaces géographiques en fonction d'évènements sociohistoriques ayant vu le jour au Québec et au Canada, comme la valorisation du français québécois dans les années 1960 et 1970.

À notre connaissance, la langue présentée aux apprenants dans les manuels de FLS n'a jamais fait l'objet de recherches concernant les représentations auxquelles elle donne lieu (pour des études portant sur l'espagnol, voir, entre autres, Arteaga, 2000 ; Monerris Oliveras, 2015 ; Wieczoreck, 1991). Toutefois, la variation stylistique, elle, a tout de même été l'objet de quelques recherches qui s'inscrivaient dans une approche variationniste (J. Auger, 2002 ; J. Auger et Valdman, 1999 ; Etienne et Sax, 2009 ; Mougeon, Nadasdi et Rehner, 2002). Il se dégage de ces recherches que les variantes appartenant au français formel sont les plus représentées dans les manuels, voire surreprésentées. En effet, les dialogues qui doivent reproduire des échanges quotidiens familiers et spontanés entre amis sont produits à l'aide de variantes formelles, alors que des variantes informelles seraient attendues (J. Auger, 2002 ; Mougeon et coll., 2002). En d'autres mots, ces recherches permettent de constater que la langue employée dans les manuels ne correspond pas entièrement à celle utilisée couramment par la population cible dans les contextes de vie réelle.

Les manuels de français ont donc fait l'objet de plusieurs études. D'une part, les études concernant les représentations s'intéressaient principalement aux représentations des apprenants et de l'apprentissage (von Münchow, 2004a) ainsi qu'à la construction culturelle (N. Auger, 2003 ; Chapelle, 2014) et n'abordaient que très peu le traitement de la langue en contexte. D'autre part, les analyses variationnistes portant sur les manuels ont permis d'établir que les usages dépeints dans les manuels n'étaient pas entièrement représentatifs des usages oraux courants de la vie quotidienne. À notre connaissance, aucune recherche n'a encore porté sur l'étude des français nationaux et de leurs locuteurs ainsi que sur les représentations qui leur sont associées dans les manuels de FLS. L'objectif de la présente étude était donc de combler cette lacune par une analyse comparée de manuels français et québécois. Pour ce faire, notre étude s'est articulée autour de deux questions de recherche :

1. Quelles sont les représentations relatives aux français nationaux et à leurs locuteurs qui sont véhiculées par les manuels de FLE et de FLS?

2. Les représentations de ces variétés diffèrent-elles en fonction du lieu d'édition des manuels? 


\section{Méthodologie}

Cette étude a adopté une approche de linguistique de discours comparative qui s'inscrivait dans une perspective sociocognitive : " une linguistique de discours se donne pour objet l'étude des fonctions de représentation et de communication du langage telles qu'elles s'actualisent à la surface des textes, dialogues et documents » (Moirand, 1992, p. 28). La méthodologie développée par von Münchow (2004a, 2004b, 2009) pour l'étude des représentations dans des manuels de L1 a été adoptée en adaptant les critères à l'objet d'étude, soit aux représentations associées aux français nationaux, aux espaces géographiques et aux locuteurs dans des manuels de FLE et de FLS. L'analyse s'est divisée en deux parties distinctes. La première consistait en une analyse descriptive comprenant les trois volets suivants : sémantique, compositionnel et énonciatif. Le premier volet consistait à répertorier les thèmes abordés ( $p$. ex. la culture, la langue [lexique, phonétique, morphosyntaxe] et l'histoire). C'est d'ailleurs dans ce volet qu'ont été consignés tous les extraits répertoriés relatifs aux français ainsi qu'aux locuteurs et aux endroits géographiques qui leur étaient associés. ${ }^{2}$ Le volet compositionnel a permis d'examiner des éléments se rapportant à la présentation matérielle du corpus comme l'organisation des chapitres, l'ordre des notions vues et l'espace accordé aux français dans chacun des manuels. Ce dernier aspect nous a permis d'observer la place occupée par le sujet dans le corpus et d'ainsi faire ressortir l'importance accordée au sujet. Le dernier volet concernait l'énonciation et il avait pour but d'interroger le rapport entre les français nationaux et les locuteurs. Il s'agissait d'étudier le positionnement que le lecteur desdits manuels pouvait inférer quant au rapport unissant ou différenciant les différentes variétés et leurs locuteurs. De plus, la mise en commun de ces indices a permis d'établir si les manuels mettaient de l'avant un rapport de force entre les variétés. C'était donc les indices discursifs répertoriés dans ce dernier volet qui ont orienté la réflexion concernant les représentations positives ou négatives qui étaient véhiculées implicitement, ou même explicitement, dans les manuels.

La seconde partie consistait en une analyse interprétative des éléments descriptifs par l'émission de réflexions se rapportant à la fonction des manuels. Il s'agissait non seulement de dégager des réflexions sur cet aspect, mais aussi de s'intéresser à l'image de la langue française ainsi qu'à celle associée à ses variétés et aux locuteurs. Pour ce faire, nous ne pouvions pas nous fier uniquement au contenu des manuels.

\section{Les manuels}

Le corpus à l'étude était composé d'un total de 10 manuels, cinq manuels français et cinq manuels québécois, tous publiés entre 2002 et 2009. Les manuels français s'adressaient à un public de grands adolescents et d'adultes suivant des cours de FLE. Ces manuels appartenaient tous à des méthodes mettant à l'avant-plan la compétence communicative en trois niveaux. Les niveaux choisis correspondaient à A2/B1 pour Tout va bien! 2 et Reflets 2 et à $\mathrm{B} 1$ pour Connexions 3, Tout va bien! 3 et Reflets 3. ${ }^{3}$ Le niveau B1 étant considéré comme étant le niveau seuil puisqu'à ce niveau, les apprenants sont jugés indépendants et fonctionnels (Alvarez, 1981). Ils sont donc capables de répondre de façon appropriée dans des situations courantes (ministère de l'Éducation nationale, s.d.), ce qui peut expliquer la raison pour laquelle ce niveau est généralement exigé pour étudier dans les pays francophones. Pour ce qui est des manuels québécois, ils s’adressaient à des adolescents ou adultes de 15/16 ans et plus, le public visé correspondait donc à des 
apprenants ayant, pour la majorité, dépassé l'âge de scolarisation obligatoire. Ils regroupaient deux manuels pour le quatrième secondaire, À propos 4 et Transmission 4 , deux manuels pour le cinquième secondaire, $A$ propos 5 et Transmission 5 , ainsi qu'un manuel conçu pour le premier cours de français au cégep, ${ }^{4}$ À mots découverts. Le choix des manuels a été fait en raison de l'année et du lieu d'édition ainsi qu'en raison de la présence marquée de français nationaux dans les divers contenus présentés. Ce qui unissait ces manuels était d'abord le public d'apprenants visé : il s'agissait d'apprenants n'ayant pas le français comme langue première et leur âge se rapportait à la fin de l'adolescence et à l'âge adulte, bien que le public cible ait été plus jeune pour les manuels québécois puisque quatre manuels sur cinq s'adressaient à des adolescents au $2^{\mathrm{e}}$ cycle de secondaire. Cette différence s'explique par la quantité très limitée ${ }^{5}$ de matériel créé pour des adultes en FLS au Québec dans la première décennie des années 2000. Le deuxième point unissant tous ces manuels était la visée communicative. Ils tendaient donc à fournir aux apprenants les connaissances nécessaires pour interagir.

\section{Le contexte}

Puisque le corpus contenait des manuels français de FLE et québécois de FLS, il apparaissait évident lors de leur consultation que les programmes desquels ils dépendaient n'étaient pas les mêmes. Pouvions-nous comparer des manuels issus de programmes éducatifs différents ? Après une analyse comparative du Cadre européen commun de référence pour les langues (CECR) pour le niveau B1 (Conseil de l'Europe, 2001) et du Programme de formation de l'école québécoise : français langue seconde du ministère de l'Éducation, du Loisir et du Sport (MELS, 2007) pour les quatrième et cinquième années du secondaire, nous avons cru qu'il était possible d'établir que les similitudes entre les deux systèmes permettaient d'effectuer une analyse probante. En effet, en raison des publics cibles, dont l'âge visé se situait à la fin de l'adolescence pour les manuels québécois, ce qui rejoignait une partie du public des manuels français (grands adolescents), de la visée communicative propre à tous les manuels ainsi que des compétences-interagir, lire et écrire-en développement partagées par les deux programmes, il était possible d'établir que les deux corpus étaient suffisamment similaires pour qu'ils puissent être comparés.

\section{Résultats}

Pour répondre à la première question, nous traiterons d'abord de la façon dont étaient présentés les variétés nationales et leurs locuteurs pour les deux espaces géographiques principalement exploités dans les manuels français et québécois, soit le Québec, puis la France. Il sera ensuite question de trois autres espaces géographiques présentés dans les manuels français, soit la Belgique, la Suisse et l'Afrique, qui seront traités simultanément. La réponse à la question deux s'ensuivra, dans laquelle les deux variétés et espaces géographiques principaux seront présentés dans le but de faire ressortir les ressemblances et différences relatives aux représentations leur étant associées.

\section{Première question de recherche}

Le Québec et le français québécois dans l'ensemble du corpus. Le Québec et le français québécois représentaient le territoire et la variété les plus souvent mentionnés, et 
ce, autant dans les manuels québécois, puisqu'il s'agissait de la variété propre au lieu d'édition des manuels, que français, lorsque la variation géographique était traitée. En effet, l'analyse du volet sémantique a révélé que le Québec était mentionné dans chacun des manuels français à l'étude en fonction de deux thèmes distincts dont le principal était la culture, regroupant des informations démographiques (Reflets 2) et historiques (Reflets 2 et Reflets 3) ainsi que le théâtre, le conte et le cinéma (Connexions 3, Tout va bien! 2 et Tout va bien! 3). Deux manuels, soit Reflets 2 et Tout va bien! 3, abordaient ces aspects dans une perspective touristique en demandant aux apprenants, par exemple, de prévoir un voyage dans un endroit francophone. Le deuxième thème abordé par tous les manuels français, à l'exception de Tout va bien! 2, était la langue parlée au Québec, thème qui se divisait en trois sous-thèmes. Le premier se rapportait au lexique (Connexions 3, Reflets 2 et Reflets 3), lequel intégrait la question des québécismes. Ensuite, le deuxième se rapportait à la langue parlée, comprenant la présentation du français oral populaire, ${ }^{6}$ qui était associé au joual (Tout va bien! 3) et l'accent (Connexions 3). Puis venait la question politique avec le bilinguisme et la loi 101 (Reflets 2) avec une mention pour le domaine de l'éducation (Reflets 2).

L'analyse compositionnelle de ces manuels à l'égard du Québec et du français parlé au Québec a révélé que très peu d'espace physique leur était réservé, soit d'aussi peu que $1 / 10$ de page à deux pages pour le manuel Reflets 2 . En effet, l'espace québécois s'est vu confiné à de petits encadrés et parfois même à une simple mention, ou il était alors traité dans un contexte plus grand, comme la Francophonie (Tout va bien! 2 et Tout va bien! 3). Des 10 occurrences répertoriées portant sur le Québec, quatre appartenaient à la section Civilisation, présentant généralement des éléments de culture générale (Reflet 2, Tout va bien! 2 et Tout va bien! 3), deux étaient liées à la section Projet en fin de module et elles servaient d'exemples à la tâche à accomplir en classe (Reflets 2 et Tout va bien! 2) et une se trouvait à être dans un encadré de phonétique (Connexions 3). En raison de leur visée culturelle et éducative, le type de support utilisé se rapportait généralement à des encadrés relatant des informations sur le Québec et sur la langue en fonction des buts didactiques et pédagogiques poursuivis par les manuels (p. ex. développement de stratégies de lecture). Deux extraits étaient tirés de documents authentiques : une chanson de Garou (Tout va bien! 2) et un article de presse (Reflets 3). De plus, les exercices associés aux supports écrits étaient majoritairement des exercices de compréhension écrite. Pour ce faire, les auteurs des manuels ont eu recours à des questions à choix multiples ainsi qu'à des questions à développement court. Deux exercices s'appuyaient aussi sur du matériel audio, soit un exercice de discrimination phonétique (Connexions 3) ainsi qu'un exercice de compréhension orale à partir d'une vidéo promotionnelle à propos de la ville de Montréal (Reflets 2). De plus, certains de ces textes et de ces exercices portaient sur la langue au Québec, ce qui nous a amenée au troisième volet de la partie descriptive : l'analyse énonciative, laquelle reposait sur les deux premiers sous-thèmes se rapportant à la langue auxquels nous avons fait référence dans l'analyse sémantique. Il sera ici question de faire ressortir les procédés textuels qui ont été mis en place pour distinguer ce qui appartenait aux français nationaux et aux usages et ce qui les distinguait.

Premièrement, trois des manuels se sont intéressés au lexique propre au français québécois. Par exemple, la Figure 1, tirée de Reflets 2 (p. 75), traite des québécismes, de variantes lexicales qui se retrouvent dans le français parlé des Québécois. Il est à noter que le lexique dit québécois est en italique et que l'équivalent est en romain. 


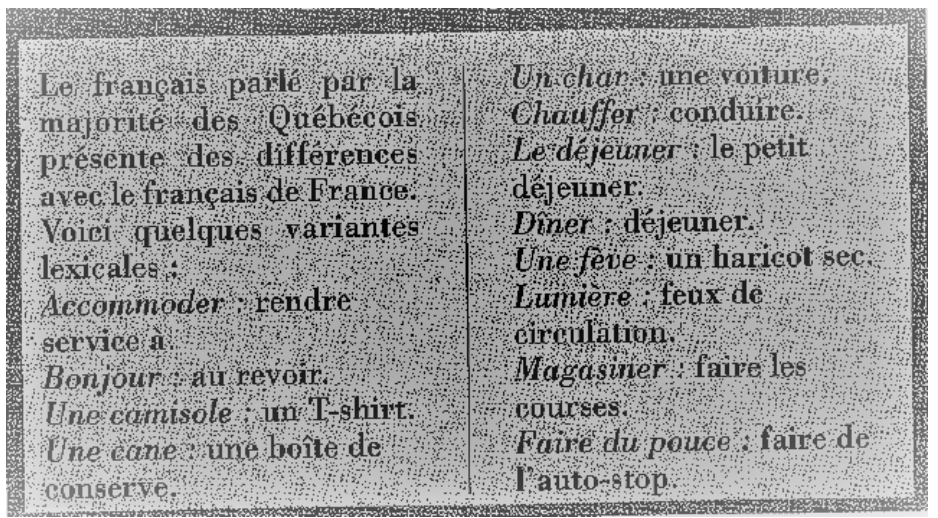

Figure 1. Le Québec est aussi. . . : la langue (Reflets 2, p. 75).

La liste des variantes comprend des verbes et des noms communs, comme souper (qui est aussi utilisé en Belgique et en Suisse), char et fève. Aucune valeur sociolinguistique et aucun contexte ne sont associés à chacun de ces mots. De plus, les définitions ne sont pas toujours adéquates. Par exemple, le mot camisole aurait pu être glosé par marcel pour donner une équivalence plus exacte, un t-shirt ayant des manches, ce qui n'est pas le cas de la camisole.

Deuxièmement, le traitement de la langue orale prenait une place importante dans les sections se rapportant au français québécois. En effet, à la page 73 du cahier de l'enseignant du manuel Reflets 2, le corrigé se rapportant à cette section indiquait que « Le français canadien se démarque du français de France non seulement par quelques différences lexicales, mais aussi par la prononciation, surtout dans le parler populaire, le joual (prononciation approximative du mot cheval) ». Tout va bien! 3 a repris des propos similaires concernant le parler populaire, ajoutant que les locuteurs auraient recours à l'emprunt de termes nautiques, par exemple : « embarquer et débarquer de voiture » (p. 68) et que les genres grammaticaux établis par les ouvrages de référence ne seraient pas toujours respectés. De plus, le guide pédagogique de Tout va bien! 3 (p. 108) a fourni des informations supplémentaires sur le français québécois, concernant la présence d'archaïsmes, de dialectalismes, de néologismes et d'emprunts à l'anglais. Dans un autre ordre d'idée, Connexions 3, avec ses exercices portant sur la phonétique, a présenté l'accent québécois (p. 121). Très peu d'explications ont été présentées quant aux particularités de cet accent, exception faite du troisième exercice proposé qui consistait à souligner les différences de prononciation entre un locuteur français et un locuteur québécois. Le guide pédagogique (p. 216) a indiqué qu'il s'agissait de la différence de prononciation de la voyelle nasale /ã/ et de l'enchainement /wa/ dans le verbe voir. En bref, d'une part, dans les manuels français, nous avons pu observer que l'exploitation du Québec et du français québécois poursuivait principalement un but culturel et un mouvement d'ouverture sur la francophonie, d'où le fait que cet aspect prenait plus d'importance que la langue utilisée sur le territoire. D'autre part, la langue orale était généralement représentée par le joual et le lexique était le sujet sur lequel les manuels fournissent le plus d'informations.

L'analyse sémantique et compositionnelle des manuels québécois à l'étude a révélé que les occurrences se rapportant au Québec, aux Québécois ainsi qu'au français québécois pouvaient être regroupées en deux thèmes principaux : la littérature et la langue. Contrairement aux manuels français, les textes sur la langue et sur le Québec se 
retrouvaient à de nombreux endroits dans les manuels, et ce, en fonction des thématiques abordées. Le premier thème exploité était la littérature ; la majorité des occurrences concernaient des extraits de textes authentiques de romans ( $\grave{A}$ propos $4, \dot{A}$ propos 5 , Transmission 5), de nouvelles ( $\grave{A}$ mots découverts) et de chroniques (Transmission 5 et $\grave{A}$ mots découverts). Le second thème était la langue, thème souvent exploité de pair avec celui de la littérature. En effet, tous les textes présentés étaient suivis d'une série d'exercices de compréhension écrite, mais certains portaient aussi sur le lexique en français québécois. À une reprise, la question du lexique n'était pas associée à la variation géographique, soit l'encadré portant sur les registres de langue dans $A$ propos 5 (p. 42-43). Dans ce dernier, le registre soutenu était associé au français littéraire écrit tandis que le registre populaire était défini comme suit : «Le vocabulaire utilisé est limité, les phrases sont souvent incomplètes et mal construites. Cette façon de s'exprimer est inacceptable à l'écrit. Ex. : Chsé pas ». L'opposition parfois réductrice entre langue orale et langue écrite était d'ailleurs présente dans tous les manuels du corpus québécois, c'est d'ailleurs sur cet aspect qu'a reposé le dernier volet analytique, l'analyse énonciative.

Dans les manuels québécois à l'étude, le travail sur la langue, plus particulièrement sur les variantes propres au français québécois, concernait principalement la question des

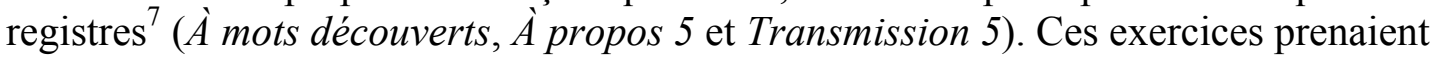
généralement place après un extrait dans lequel la langue orale était mise de l'avant, comme c'était le cas dans une entrevue ou dans une pièce de théâtre. Par exemple, dans un texte du chroniqueur Bruno Blanchet (Transmission 5, p. 32-34), on a demandé aux apprenants de donner l'équivalent de l'expression familière «C'tu drôle hein? », en italique dans le texte, en français standard. De plus, deux manuels traitaient de ce qui était désigné comme étant des anglicismes (mais qui auraient plutôt dû être catégorisés de québécismes par rapport au français standard ; À mots découverts et Transmission 5), demandant aux apprenants de trouver l'équivalent en français. Il est d'ailleurs à noter que tous ces anglicismes se trouvant dans le texte étaient en italique afin de les différencier du restant du texte, qui était en romain. Toutefois, la différenciation du français québécois par rapport à celui de France n'était présentée explicitement que dans deux manuels. Le premier était Transmission 5 dans lequel un texte portant sur le Québec et sa culture stipulait que «notre langue française est différente de celle de la France et de tous les autres français du monde » (p. 118), affirmant ainsi sa différence. Le second manuel, À mots découverts, présentait un plus grand nombre d'occurrences statuant de cette différence. En effet, les québécismes étaient tous identifiés dans un glossaire à même les textes, il en allait de même avec les anglicismes, comme swigner, fun et reeler (p. 40-44), qui étaient identifiés comme tels par les auteurs du manuel. De plus, ces encadrés contenaient aussi des informations relatives aux registres, aux tournures orales, aux réalisations orales ainsi qu'aux emprunts à d'autres langues. Globalement, on demandait aux apprenants de reconnaitre ces variantes et de les associer au bon registre. De plus, pour des mots associés aux registres plus informels comme les registres vulgaire et populaire, on demandait de trouver leur équivalent dans un français standard. La variation lexicale propre au français québécois dans $\grave{A}$ mots découverts était donc majoritairement représentée par la langue orale et des variantes informelles, exception faite de soccer (p. 40-44) et de courriel (p. 66-73), soit deux occurrences sur une cinquantaine de pages de textes et d'exercices. En ce qui concerne la variation sur un plan régional, la question des accents régionaux était abordée une seule fois dans $A$ A propos 5 (p. 51), mais il s'agissait d'un prétexte pour traiter de la diversité des entreprises au Québec, la question de la langue n'était donc pas abordée directement. 
La France et le franco-français dans l'ensemble du corpus. Pour sa part, la France occupait une grande partie des manuels français, comme mentionné précédemment. L'analyse sémantique du sujet nous a permis d'observer que, comme pour le traitement du territoire québécois, l'ensemble du matériel portant sur la France et sur la langue française pouvait aussi se diviser en deux thèmes principaux. Le premier étant la culture, nous avons retrouvé des sujets comme les arts et la musique (Reflets 2, Reflets 3, Tout va bien! 2 et Tout va bien! 3) ainsi que la politique (Reflets 2 et Reflets 3). Deux de ces manuels traitaient de la France sous un aspect plus touristique, comme c'était le cas dans Reflets 2, manuel dans lequel une région francophone, de France ou d'ailleurs, était présentée à la fin de chacune des unités dans la section Civilisation. Le second thème abordé était celui de la langue, qui se divisait en quatre sous-thèmes : la phonétique (Connexions 3 et Tout va bien! 3), la langue parlée qui était associée au verlan (Tout va bien! 3), les emprunts historiques (Reflets 3) ainsi que les expressions figées (Connexions 3). L'analyse compositionnelle des manuels a révélé que, comme pour le traitement du Québec et de sa langue, il s'agissait de sections assez restreintes, de 2 pages (Reflets 2) à un petit encadré prenant le 1/6 de la page (Connexion 3). De plus, la majorité de ces informations se situait dans la section intitulée Civilisation et se retrouvait dans des textes informatifs auxquels un questionnaire était annexé. L'analyse énonciative a permis d'établir que l'accent était principalement mis sur le lexique propre au français standard, comme la question des registres qui était abordée dans Tout va bien! 3 (p. 67). De plus, dans Reflets 3 (p. 146-147) et dans Tout va bien! 3 (p. 67), il était question d'emprunts, comme charabia et baragouiner, de mots intégrés puisque présents et définis dans les ouvrages de référence et dont l'intégration linguistique est expliquée par des évènements historiques. Dans un autre ordre d'idée, la question de langue, patois, dialecte, jargon et argot était aussi abordée dans l'extrait présenté à la Figure 2 (Tout va bien! 3, p. 67). Aucune définition de ce que pouvait constituer la différence entre langue, patois, dialecte, jargon et argot n'était fournie. De plus, il y avait mention de mots dits avec un accent, mais l'accent n'était pas spécifié. En fait, si l'on se fiait à la consigne au bas de l'extrait (voir Figure 2), cet encadré servait d'introduction à une discussion abordant la question de la variation géographique, mais pas celle de la langue française.

\section{Ronde des mots}

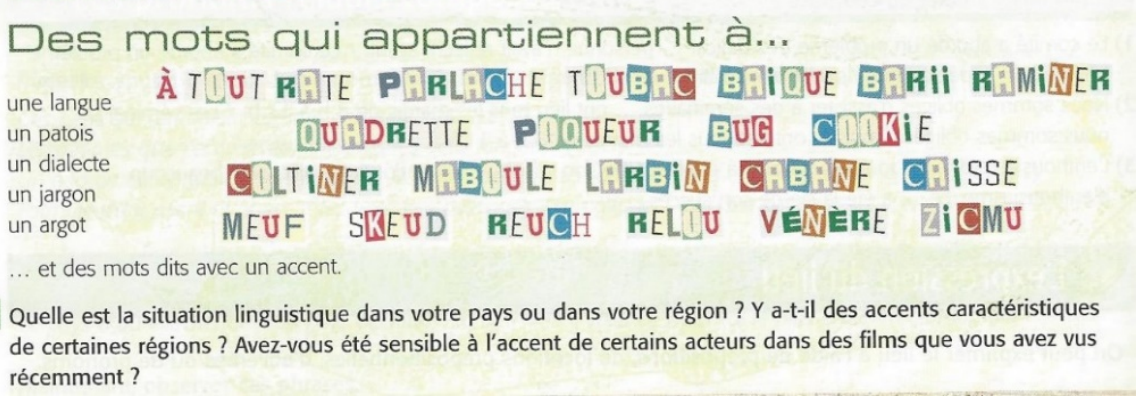

Figure 2. Ronde des mots (Tout va bien! 3, p. 67).

La variation géographique du français n'était donc que très peu traitée, mais les occurrences trouvées concernaient la langue orale selon deux éléments : le verlan et l'accent. Le verlan 
était présenté dans Tout va bien! 3, aux côtés du joual et du français d'Afrique, comme étant le parler des jeunes utilisé dans les pays francophones européens.

La question de l'accent était traitée, comme pour l'accent québécois, dans le manuel Connexions 3 (p. 107), sous l'angle de la variation régionale au sein de la France. En effet, l'encadré sur la phonétique s'intéressait aux accents régionaux, et spécifiquement deux accents du sud de la France, l'accent marseillais et celui de la région de l'Aveyron, ainsi que ceux de la Corse et de l'Alsace. Des informations complémentaires sur le sujet des accents régionaux se trouvaient dans le guide pédagogique (p. 189) : " Même si le "français parisien" (ou "français standard") tend à s'imposer un peu partout en France, à travers les médias en particulier, il reste encore possible d'entendre des prononciations particulières dans certaines régions françaises » et « Les prononciations du sud de la France sont celles qui résistent le plus à l'avancée de la prononciation "standard". Une comparaison est proposée entre les deux prononciations ». Le vocabulaire utilisé pour traiter de la présence des différents accents semblait indiquer un rapport de force entre la langue du Nord et celle du Sud.

Pour leur part, les manuels québécois ne laissaient que très peu de place à la France, à l'exception de la présence de textes littéraires de genres différents : poésie, théâtre et roman (A propos 4 et $\dot{A}$ propos 5 ). Les auteurs des textes présentés étaient, pour la plupart, identifiés par leur nom ainsi que par leur pays d'origine. Parmi les textes présentés dans la section Lecture de ces deux manuels, un seul était d'origine québécoise dans $\grave{A}$ propos 4 et deux textes avaient des auteurs canadiens, une Néo-Brunswickoise et un Québécois dans $\grave{A}$ propos 5 . Tous les autres textes étaient français. Toutefois, aucune mention des particularités du français de France n'était présente, et ce, autant pour la langue orale qu'écrite. Bien que la variation géographique n'ait pas été traitée, le territoire français était mentionné dans le manuel Transmission 5 lorsqu'il était question d'histoire, plus précisément de la colonisation du territoire.

La Belgique, la Suisse, l'Afrique et les français d'ailleurs. La Belgique était présente dans deux des manuels français, soit Reflets 2 et Tout va bien! 3, dans lesquels les textes présentaient les attraits touristiques ainsi que la coexistence de trois langues officielles sur le territoire. De plus, la langue française était aussi un thème abordé. En effet, quelques belgicismes ont été présentés dans Reflets 2 (p. 107) tels que nonante, sacoche, carte-vue et bas à ordure. De plus, le verlan était aussi associé à la Belgique (Tout va bien! 3) puisque, comme indiqué à la page 68 du manuel, il est utilisé dans les pays francophones européens, alliant ainsi la France, la Belgique et la Suisse. Cette dernière était d'ailleurs mentionnée dans Tout va bien! 2 par la transcription d'une tradition orale suisse (p. 138) et de la mention de septante-six pour soixante-seize dans un extrait audio, dont le texte était retranscrit dans le manuel à la page 46. Enfin, le français en Afrique était présenté dans deux manuels, soit Connexions 3 et Tout va bien! 3. Le matériel fourni avec le premier manuel permettait aux apprenants d'entendre des accents ivoirien et sénégalais, proposant ainsi une variation au sein du continent, tandis que le deuxième manuel traitait de la notion de « gros mots » en français d'Afrique, proposant ainsi des usages similaires sur tout le continent.

Pour conclure la réponse à la première question de recherche, revenons sur le fait que différentes variétés de français étaient présentées dans les manuels, bien que leur traitement eut été souvent bref et limité, soit les français de France, du Québec, de la Belgique, de la Suisse et de l'Afrique, mais les variétés étaient représentées de façon très 
différente. En effet, le français du Québec était généralement représenté par des variantes ou des traits dits informels et souvent stigmatisés, et ce, autant dans les manuels français que québécois. Ensuite, la variation en France, non traitée dans les manuels québécois, était principalement associée au verlan, tout comme les autres français européens. Enfin, le français d'Afrique, par son appellation, était présenté comme étant homogène sur l'ensemble du continent, sauf dans l'exercice de phonétique tiré de Connexions 3. Malgré ces différences, un point commun unissait tous les manuels composant le corpus, il s'agissait de la présence marquée de la langue standard, généralement opposée aux usages courants et oraux. Les manuels étudiés s'inscrivaient donc tous dans une tradition de conformisme linguistique qui se rapportait à une « représentation idéalisée de la langue française basée sur la langue écrite et la tradition graphique conservatrice, peu encline à la tolérance et à la variation » (Jejcic, 2008, p. 220). De plus, tel qu'il a été mentionné dans la partie traitant du français québécois dans les manuels du Québec, la plupart des exercices qui traitaient des variantes québécoises demandaient aux apprenants de les transposer dans une langue dite standard.

\section{Deuxième question de recherche}

Ayant préalablement établi la façon dont les différentes variétés de français ainsi que la variation géographique étaient traitées dans les corpus de manuels à l'étude, nous pouvons maintenant répondre à la deuxième question de recherche en établissant les différences entre les représentations selon l'origine des manuels. Il sera d'abord question de la présentation du français de France et du territoire, puis de celle du français du Québec et du territoire et, enfin, de celle des autres variétés étudiées, soit les français d'Europe et d'Afrique.

Tout d'abord, la présentation du français de France différait d'un sous-corpus à l'autre, notamment par le type de matériel utilisé pour illustrer la France et sa langue, ce qui amène un traitement différencié de la question. D'une part, $A$ propos 4 et $A$ propos 5 abordaient le sujet de la France par la littérature, généralement par des textes classiques, dans lesquels la langue orale était sous-représentée. L'exception était une pièce de théâtre de Molière (Á propos 5, p. 106-111), mais les usages n'étaient pas ceux typiques du français parlé contemporain et n'étaient pas représentatifs de la langue courante utilisée en contexte informel ou même formel. De plus, les exercices portant sur le vocabulaire des textes traitaient de mots courants et utilisés autant en France qu'au Québec. Le français utilisé en France n'était donc pas présenté comme étant différent, mais plutôt comme étant un élément partagé par les deux endroits. La seule mention de la différence entre les deux français (Transmission 5, p. 118) ne permettait pas de déterminer précisément en quoi les usages différaient, il était uniquement indiqué que les différences étaient d'origine historique. Du côté des manuels français, Tout va bien! 3 était le seul manuel qui abordait la question de « la langue parlée qui a donné lieu à des appropriations très locales » (p. 68), notamment par le recours au verlan en France en stipulant qu'il s'agissait d'un argot parlé par les jeunes. Selon cet extrait, c'était par la langue parlée que les français nationaux se différenciaient, mais il existait aussi une variation possible au sein d'un même territoire, comme c'était le cas pour l'exercice de phonétique dans Connexions 3. Dans cet exercice, la variation prenait le visage de l'accent qui n'était pas celui du Nord ou celui dit de Paris (voir Connexions 3, p. 107), qui était considéré comme étant le standard, comme indiqué dans le guide pédagogique du manuel (p. 216). Bien que l'on ait retrouvé davantage 
d'éléments se rapportant au traitement de la variation géographique dans les manuels français, c'était les différences des autres variétés qui étaient mises de l'avant. En effet, la grande majorité des éléments présentés concernaient des aspects (archaïsmes, dialectismes, accents, néologismes, parlers populaires) qui n'étaient pas associés à la variété nationale, présentant ainsi le français de France comme étant le point de comparaison. Le discours était donc associé au regard de la France, pays d'édition des manuels, sur les autres variétés.

C'était d'ailleurs cet aspect qui différenciait le plus les manuels français des manuels québécois puisque ces derniers ne traitaient que de la variation au sein du français québécois de façon explicite. Elle était bien sûr la variété à l'étude puisque propre au territoire géographique et à son public cible, mais elle ne servait pas de point de comparaison aux autres français. Ce phénomène s'observait plus particulièrement dans un des manuels à l'étude, soit $\hat{A}$ mots découverts, dans lequel le travail sur la langue portait exclusivement sur le français québécois : les exercices sur le lexique ou sur les expressions orales présentées dans les différents textes attestaient des usages des Québécois en contexte informel, et ce, autant pour ce qui était des éléments lexicaux, phonétiques que morphosyntaxiques. Puisque ces éléments étaient généralement présentés dans des textes, le contexte de présentation pouvait aider les apprenants à comprendre et à reconnaitre les différents usages. Dans les manuels français, seulement deux aspects de la variation propre au Québec étaient présentés, soit la variation lexicale et phonétique, mais l'espace dédié au Québec et à sa langue était assez restreint. Pour ce qui était du traitement de la variation lexicale, les mots étaient généralement présentés dans un glossaire, sans contexte d'utilisation et sans explication métalinguistique qui aurait pu fournir des informations pertinentes concernant leur emploi dans des situations appropriées et la valeur sociolinguistique de ces traits. De plus, les informations présentées dans le guide pédagogique de Tout va bien! 3 (p. 108) traitaient des archaïsmes qui auraient été présents dans la langue québécoise, comme niaiseux et po[i]gner, exposant ainsi une vision ethnocentrée de la langue puisque ces mots sont bel et bien vivants au Québec. L'étude des exemples présentés précédemment a démontré que la vision du français québécois n'était pas des plus positives puisque les éléments mentionnés se rapportaient surtout à des éléments associés au français populaire (même si ce n'était pas toujours explicitement indiqué), comme le joual, les mots comme char et la présence courante de termes étant désignés comme étant des anglicismes. En fait, il n'était mentionné nulle part à quoi ressemble le français québécois utilisé dans d'autres contextes.

En ce qui concerne les variétés de français européennes, la variation, selon les exemples présentés, aurait principalement été de nature orale et, bien que ces variantes n'aient pas été comparées entre elles, certaines étaient partagées par la Belgique et par la Suisse. De plus, l'emploi de certaines de ces variantes était aussi partagé par les locuteurs québécois, comme souper et faire la file, mais cette information était manquante. De plus, aucune mention n'était faite des accents belges et suisses. La variation sur le territoire africain était, elle aussi, très peu exploitée. D'une part, le manuel Connexions 3 traitait d'une situation hétérogène sur le continent par la présence de différents accents. D'autre part, Tout va bien! 3 présentait une tout autre situation : l'appellation de français d'Afrique donnait l'impression au lecteur que les usages en Afrique étaient partagés par l'ensemble des locuteurs présents sur le continent. Dans ce cas-ci, la langue n'était pas seulement une unité nationale, mais continentale. Non seulement les informations étaient peu nombreuses, 
mais elles véhiculaient une image non représentative de la réalité linguistique et politique du continent.

En bref, l'étude des représentations des français nationaux et des locuteurs dans le corpus de manuels précédemment présenté a mis en lumière le fait que les usages n'étaient pas dépeints de façon objective, et ce, autant en ce qui concernait la variété propre au lieu d'édition des manuels que les autres. D'une part, les manuels québécois traitaient très peu des autres variétés, et lorsque c'était le cas, c'était par l'intermédiaire de la littérature française. Les éléments se rapportant uniquement au français québécois étaient généralement associés à un registre plutôt informel et c'était les traits socialement marqués qui étaient majoritairement mis de l'avant. Ces éléments ne mettaient pas de l'avant l'image d'une langue dynamique et plurielle. D'autre part, les manuels français établissaient un rapport de force entre les français nationaux puisque le point de comparaison était globalement la variété française, alors appelé français de Paris, et ce, même au sein du territoire.

\section{Discussion}

Le but de cette étude était de déterminer, d'une part, la façon dont étaient présentés les variétés nationales de français, les locuteurs ainsi que les espaces géographiques leur étant associés et, d'autre part, de comparer les représentations afin de faire ressortir les différences en fonction du lieu d'édition des manuels. Afin de répondre à ces deux questions, nous avons eu recours à une méthode d'analyse de manuels s'inscrivant dans la linguistique de discours comparative (von Münchow, 2004a, 2009), laquelle repose sur une analyse en deux temps : l'analyse descriptive, avec les trois volets d'analyse sémantique, compositionnel et énonciatif, suivie de l'analyse interprétative. L'analyse descriptive nous a permis de recenser toutes les occurrences traitant des français nationaux et de leurs locuteurs, selon les thèmes abordés, l'espace et l'organisation physique de ces extraits et les éléments implicites et explicites se rapportant aux ressemblances et différences entre les français nationaux. L'analyse interprétative nous a ensuite permis de faire ressortir les différences sur le plan du traitement des variétés nationales et des locuteurs.

L'analyse des manuels a fait ressortir le fait que les représentations répertoriées différaient en fonction du lieu d'édition des manuels (il ne faut d'ailleurs pas négliger l'influence des auteurs ayant travaillé à l'élaboration de ces manuels, de par la tradition académique et didactique dans laquelle ils s'inscrivaient). En effet, bien que le traitement de la variation ait semblé se manifester par le lexique et par la langue dite orale, et ce, autant dans les manuels français que québécois, les représentations qui en ont découlé n'étaient pas les mêmes. Dans les manuels québécois, la seule variété nationale présentée explicitement était le français québécois, la France n'étant représentée que de façon implicite par la présence d'auteurs de textes littéraires. Il en ressort donc que les manuels québécois à l'étude contribuaient peu à l'éveil à la variation géographique, comme entendu par Bachman (1990, 2010). En ce qui concerne la seule variété présentée de façon explicite, le français québécois, son traitement passait exclusivement par le lexique, notamment par des exercices de transposition d'un registre à l'autre ( $A$ mots découverts), et par les emplois associés à la langue orale en contexte informel (registre populaire). Ces résultats font écho à ceux de J. Auger (2002) qui a fait ressortir que, lorsqu'il était question du français

québécois dans les manuels utilisés dans des classes d'immersion, seul le lexique était traité. Ce traitement de la langue, dans lequel on demandait aux apprenants de transposer 
les québécismes en langue standard, pouvait influer sur la perception qu'avaient les apprenants de la variété québécoise. En effet, il serait possible d'inférer, du point de vue du lecteur ou de l'apprenant, que les particularités linguistiques se rapportant à la variété québécoise étaient non standard et stigmatisées.

Cette observation nous amène d'ailleurs à traiter du métalangage employé pour traiter de la langue dans les manuels à l'étude. Les phénomènes décrits et associés à des variétés précises de français nationaux n'étaient pas toujours désignés adéquatement, puisque leur catégorisation était parfois imprécise ou même erronée. Par exemple, le métalangage employé pour classifier les différents phénomènes linguistiques amenait le lecteur à hiérarchiser les traits lexicaux présentés, sans nécessairement tenir compte du contexte d'énonciation, comme c'était le cas dans le type d'exercice sur les registres mentionné précédemment. Puisqu'il a été établi que les frontières entre les registres sont plutôt floues et que les usages des locuteurs natifs empruntent à tous les registres (voir notamment Wachs, 2005), il serait intéressant de classifier différemment les variantes en fonction de leur contexte d'énonciation plutôt qu'en fonction des catégories abstraites que sont les registres. Il serait aussi pertinent d'indiquer plusieurs variantes présentes sur un territoire géographique donné afin de donner une meilleure idée des usages réels des locuteurs. Un autre élément qui a attiré notre attention était la question des anglicismes lorsque le français québécois était abordé. En fait, certains mots désignés dans les manuels comme étant des anglicismes devraient plutôt être classifiés de québécismes, et ce, pour deux raisons. En premier lieu, ces « anglicismes » sont exclusivement utilisés au Québec (et certains au Canada) et sont donc partagés par un ensemble de locuteurs restreints sur un territoire donné. En second lieu, comme avancé par Paquet-Gauthier (2015) pour les unités lexicales perçues comme étant des emprunts sémantiques à l'anglais, il est très difficile de démontrer avec certitude que le sens que prend un mot provient réellement de l'anglais plutôt que d'être le produit d'un consensus sémantique à l'intérieur d'une communauté de pratique. De plus, le recours au terme anglicisme tend à avoir un aspect dépréciatif et une connotation péjorative (Courbon et Paquet-Gauthier, 2014), ce qui contribue implicitement à la dépréciation de la langue et à la transmission de représentations négatives.

Bien qu'il ait été établi que le traitement des représentations associées aux français nationaux relatif au développement de la compétence sociolinguistique n'avait jamais fait l'objet de recherches, nos résultats font tout de même écho aux résultats des études variationnistes concernant le traitement des variantes stylistiques dans les manuels (J. Auger, 2002 ; J. Auger et Valdman, 1999 ; Etienne et Sax, 2009 ; Mougeon et coll., 2002). Il nous est donc possible de conclure que la représentation des français nationaux était incomplète dans les manuels québécois puisque son traitement était minoritaire dans les manuels, que les informations étaient souvent incomplètes, que des raccourcis parfois réducteurs ont été pris pour traiter de la variation lexicale et que la variation était majoritairement représentée par des traits socialement marqués. En ce qui concerne les manuels français, il s'agissait de manuels qui intégraient plusieurs français nationaux à leur contenu, contrairement aux manuels québécois. La présentation de différentes variétés de français et des lieux géographiques où elles sont parlées permettrait d'initier les apprenants au sujet et ainsi développer un éveil à la variation, comme présenté par Bachman (1990, 2010), soit de les rendre conscients que plusieurs variétés d'une langue existent dans le monde (français nationaux) et que plusieurs variétés peuvent même coexister sur le même territoire (français régionaux ; Connexions 3, A propos 5). Il est toutefois important de mentionner que, globalement, le traitement des variétés était superficiel. Bien que la 
conscientisation au phénomène ait été possible par les contenus abordés pour les manuels édités en France, la présentation n'était pas entièrement objective, notamment lorsqu'il était question de définir ce qu'était la langue standard, ici associée à un espace géographique (p. ex. Connexions 3, p. 189). On a noté un rapport de force entre la forme standard et les accents du Sud, comme quoi ce dernier n'était pas standard, bien que représentatif de la région.

La question du standard est d'ailleurs difficile à définir, comme le démontre la présence ou l'absence de guillemets accompagnant ce terme dans Connexions $3: \ll 3 . b$ ) Écoutez et comparez l'accent de Marseille que vous venez d'entendre avec une prononciation "standard” » (p. 107) et « Comparez les phrases prononcées en français standard et en français du Québec. Soulignez les parties des mots prononcés différemment » (p. 121). L'emploi des guillemets n'était aucunement expliqué : s'agissait-il de guillemets ironiques ? Servaient-ils à protéger les locuteurs et les apprenants qui étaient dans le sud de la France ? Ces extraits démontrent que les français nationaux étaient hiérarchisés et cette représentation pouvait être transmise aux apprenants par les manuels. En bref, tous les manuels du corpus présentaient des lacunes concernant le traitement des français nationaux et puisque le manuel sert souvent d'outil ou de support à l'apprentissage, il serait pertinent que ce manque soit adressé, comme l'ont fait Detey, Durand, Laks et Lyche (2010) avec la présentation de matériel diversifié et documenté concernant de nombreuses variétés de français, ce matériel était destiné à des enseignants de français (langue première, langue seconde ou langue étrangère).

En effet, les représentations sur la langue et sur celle de l'Autre influencent l'acquisition au sens où, comme avancé par Preston (2013), il existe une corrélation entre les représentations associées à la langue cible et la compréhension et la discrimination des différentes variétés. C'est sur cet aspect de l'acquisition que peuvent influer les manuels, si des explications objectives sont présentées aux apprenants. Pour ce faire, il serait pertinent que les équipes travaillant sur la conception des manuels soient composées d'experts de différents domaines (sociolinguistes, phonéticiens, didacticiens, éditeurs, etc.), tel qu'il a été soulevé par Beaulieu, French et Gagnon (sous presse). De plus, afin de compléter les informations fournies dans le matériel scolaire et de fournir un enseignement informé et objectif sur cet aspect de la langue, il est nécessaire de combler les lacunes présentes dans les manuels du corpus. Il est d'ailleurs possible de le faire par divers moyens, notamment par l'enseignement et par le recours à des corpus oraux colligeant des données sur l'ensemble de la Francophonie et parfois même proposant du matériel pour enseignants (p. ex. Le projet Phonologie du français contemporain). Il est d'ailleurs possible d'exploiter ces corpus oraux en salle de classe afin que les apprenants se familiarisent avec les usages en contexte, tel qu'il a été démontré par Bardovi-Harlig, Mossman et Vellenga (2015).

\section{Conclusion}

Cette étude a révélé que les manuels formant le corpus avaient tendance à partager une vision souvent stéréotypée des usages des locuteurs en raison d'explications incomplètes, voire parfois même erronées, se rapportant aux différentes variantes, de désignations inexactes relativement aux phénomènes décrits et du fait que les traits socialement marqués étaient surreprésentés par rapport aux variantes neutres ou formelles, et ce, peu importe la variété ayant été mise de l'avant. La place accordée au sujet était minime, les explications étaient trop peu nombreuses et elles étaient souvent incomplètes et 
l'opposition entre langue dite orale et écrite était rapide et réductrice. Le manque d'informations complètes, justes et objectives jouait un rôle primordial concernant les représentations véhiculées aux apprenants et lecteurs de ces manuels. Cette présentation des variétés pouvait donc grandement influencer les représentations des apprenants à l'égard des variétés de la langue cible. Malgré les avancées et le nombre croissant de recherches sur le sujet, les résultats ont indiqué qu'il serait pertinent de poursuivre la recherche sur la question dans le but de non seulement sensibiliser les apprenants à la variation, mais aussi de leur fournir les connaissances nécessaires au développement d'une opinion avisée.

Somme toute, cette étude de manuels n'était pas sans limites, notamment en ce qui concerne le choix des manuels composant le corpus. Tout d'abord, les manuels n'étaient pas récents, puisque, comme mentionné précédemment, plusieurs manuels avaient été édités dans les 4 dernières années, dont deux méthodes adaptées au contexte nordaméricain. Il serait donc intéressant de poursuivre l'analyse avec un plus grand nombre de manuels récents afin de voir si les représentations répertoriées dans la présente étude sont les mêmes. Dans un autre ordre d'idée, l'une des limites principales concernant ce type d'analyse, selon Moirand (1992), serait l'influence de la subjectivité de l'auteur. Il est possible que la variété de français qui définit notre appartenance linguistique et culturelle ait influencé l'analyse faite précédemment concernant les représentations, bien qu'elles reposent sur des éléments observables dans les manuels. De plus, le fait de mieux connaître le contexte de la langue au Québec qu'en France peut engendrer une réflexion moins complète pour les manuels français.

Malgré ses limites, cette étude contribue tout de même à l'avancement des connaissances relatives au développement de la compétence communicative. Plus précisément, notre contribution à l'avancée des connaissances relatives au développement de la compétence sociolinguistique telle que conçue par Bachman (1990, 2010) est d'avoir une meilleure compréhension du développement de la compétence sociolinguistique et de documenter le traitement des français nationaux, des espaces géographiques et des locuteurs, ce qui à notre connaissance n'avait pas encore été fait.

La correspondance devrait être adressée à Marie Duchemin.

Courriel : marie.duchemin.1@ulaval.ca

\section{Notes}

${ }^{1}$ L'appellation de français nationaux renvoie à la définition donnée par Hausmann (1986) selon laquelle « de par leur souveraineté nationale, ces unités géolinguistiques [Québec, Belgique, Suisse, etc.] méritent la dénomination de pays tout autant que la France. Les particularités lexicales de ces pays par rapport au français de France ne sont donc pas des régionalismes. Ils ne constituent pas une variante régionale de la langue française mais une variante nationale » (p. 4). Les français nationaux comprennent la norme nationale ainsi que les variations régionales et sociales propres à chacune des unités géolinguistiques.

${ }^{2}$ Bien que nous traitions avant tout des français nationaux, il existe une forte corrélation entre ce que les individus pensent de la langue et leurs attitudes et croyances envers les locuteurs (Preston, 2013). Il est donc difficile de différencier les représentations associées à 
la langue et celles relatives aux locuteurs, c'est pourquoi nous avons choisi de les intégrer dans notre analyse des représentations sur la langue (voir aussi N. Auger, 2003).

${ }^{3}$ Afin de ne pas alourdir le texte, les manuels seront dénommés par leur titre, les notices bibliographiques complètes se trouvant dans les références des manuels composant le corpus.

${ }^{4}$ Le cégep, institution fréquentée après le secondaire, propose des formations préuniversitaires et professionnelles.

${ }^{5}$ La méthode Echo pour l'Amérique du Nord (Callet et coll., 2015) ainsi que la méthode Par ici (Desjardins, Sauvé et Usereau, 2015) sont parues après la sélection des manuels composant le corpus.

${ }^{6}$ Dans le cadre de cet article, le terme français populaire se rapporte à la définition proposée par Gadet (1992) : « Selon une opinion largement partagée, il existe, à côté de la langue française standard, objet de la plupart des descriptions grammaticales, un "français populaire" ayant ses traits linguistiques propres, une capacité à organiser la signification, et qui serait parlé par les couches sociales défavorisées » (p. 3).

${ }^{7}$ Selon Wachs (2005), il s'agit « [d]es différents “styles” dont dispose un locuteur pour s'exprimer, c'est-à-dire le fait inexorable qu'il fasse varier sa langue en fonction de la situation de communication. Il s'agit de la variation stylistique » (p. 169). Elle indique aussi que le traitement des registres se trouve souvent à être réduit au lexique, notamment dans les manuels de langue.

\section{Références}

Alvarez, G. (1981). Niveau-seuil et enseignement fonctionnel du français. Québec français, $42,33-35$.

Arteaga, D. L. (2000). Articulatory phonetics in the first-year Spanish classroom. Modern Language Journal, 84(3), 339-354.

Auger, J. (2002). French immersion in Montreal: Pedagogical norm and functional competence. Dans S. Gass, K. Bardovi-Harlig, S. Sieloff Magnan et J. Walz (dir.), Pedagogical norms for second and foreign language learning and teaching (p. 81101). Philadelphie, PA : John Benjamins.

Auger, J. et Valdman, A. (1999). Letting French students hear the diverse voices of Francophony. The Modern Language Journal, 83(3), 403-412. doi : 10.1111/00267902.00030

Auger, N. (2003). Les représentations interculturelles dans des manuels de français langue étrangère en usage dans l'Union européenne. Dans M.-C. Alén Garabato, N. Auger, P. Gardies et E. Kotul (dir.), Les représentations interculturelles en didactique des langues-cultures : enquêtes et analyses (p. 35-71). Paris, France : L'Harmattan.

Bachman, L. (1990). Fundamental considerations in language testing. Oxford, RoyaumeUni : Oxford University Press. 
Bachman, L. (2010). Fundamental considerations in language testing. Oxford, RoyaumeUni : Oxford University Press.

Bardovi-Harlig, K., Mossman, S. et Vellenga, H. E. (2015). Developing corpus-based materials to teach pragmatic routines. TESOL Journal, 6(3), 499-526.

Beaulieu, S., French, L. et Gagnon, S. (sous presse). I'veulent-tu parler comme nous-autres ? Opinions d'apprenants sur la forme interrogative totale en français laurentien. Arborescences.

Callet, S., Gibbe, C., Girardet, J., Liakin, D., Liakina, N., Olivry, F. et Pécheur, J. (2015). Echo A2-Méthode de français pour l'Amérique du Nord. Paris, France : CLE International.

Chapelle, C. A. (2014). Five decades of Canadian and Québec content in French textbooks in the United States. American Review of Canadian Studies, 44(4), 415-432.

Chevrot, J.-P., Chabanel, D. et Dugua. C. (2007). Pour un modèle de l'acquisition des liaisons basé sur l'usage : trois études de cas. French Language Studies, 17, 103 128. doi : $10.1017 / \mathrm{S} 0959269506002663$

Chiss, J.-L. et Cicurel, F. (2005). Présentation générale : cultures linguistiques, éducatives et linguistiques. Dans J.-C. Beacco, J.-L. Chiss, F. Cicurel et D. Véronique (dir.), Les cultures éducatives et linguistiques dans l'enseignement des langues (p. 1-9). Paris, France : Presses Universitaires de France.

Conseil de l'Europe. (2001). Cadre européen commun de référence pour les langues : apprendre, enseigner, évaluer. Repéré à http://www.coe.int/T/DG4/Linguistic/Source/Framework_FR.pdf

Courbon, B. et Paquet-Gauthier, M. (2014). Faux amis/vrais ennemis : réutilisations de la notion d'anglicisme dans le discours métalinguistique au Québec. Le discours et la langue, 6(1), 143-173.

Desjardins, N., Sauvé R. et Usereau, M. (2015). Par ici : méthode de français. Montréal, QC : Les Éditions MD.

Detey, S., Durand, J., Laks, B. et Lyche, C. (dir.). (2010). Les variétés du français parlé dans l'espace francophone. Ressources pour l'enseignement. Paris, France : Ophrys.

Etienne, C. et Sax, K. (2009). Stylistic variation in French : Bridging the gap between research and textbooks. The Modern Language Journal, 93(4), 584-606.

Gadet, F. (1992). Le français populaire (Coll. Que sais-je ?). Paris, France : PUF.

Hausmann, F. J. (1986). Les dictionnaires du français hors de France. Dans L. Boisvert, C. Poirier et C. Verreault (dir.), La Lexicographie québécoise : bilan et perspectives : actes du colloque organisé par l'équipe du Trésor de la langue française au Québec et tenu à l'Université Laval les 11 et 12 avril 1985 (vol. 8, p. 3-21). Québec, QC : Presses de l'Université Laval.

Hymes, D. (1972). On communicative competence. Dans J. B. Pride et J. Holmes (dir.), Sociolinguistics: Selected readings (p. 269-293). Harmondsworth, RoyaumeUni : Penguin.

Jejcic, F. (2008). Pratiques de l'écrit et perception de la norme : une enquête en France et au Canada en 2002. Dans B. Horiot (dir.), Français du Canada-Français de France : actes du septième colloque international de Lyon. 16-18 juin 2003. Tübigen, Allemagne : Niemeyer.

Ministère de l'Éducation nationale, de l'Enseignement supérieur et de la Recherche. (s.d.). CECRL : le Cadre européen commun de référence pour les langues. Repéré à 
http://eduscol.education.fr/cid45678/cadre-europeen-commun-de-referencececrl.html

Ministère de l'Éducation, des Loisirs et du Sport (MELS), Gouvernement du Québec. (2007). Programme de formation de l'école québécoise : français langue seconde. Repéré à http://www1.mels.gouv.qc.ca/sections/programmeFormation/secondaire $2 /$ medias/en 15c_QEP_FLS_FrancBase.pdf

Moirand, S. (1992). Des choix méthodologiques pour une linguistique de discours comparative. Langages, 26(105), p. 28-41.

Monerris Oliveras, L. (2015). Spanish dialectal variation in the foreign language classroom: Students' attitudes, instructors' beliefs and teaching practices, and treatment of variation in textbooks (Thèse de doctorat inédite). Université d'Alberta, Calgary, AB. Repéré à https://era.library.ualberta.ca/files/pz50gz965/Monerris_Oliveras_Laura_201412_P hD.pdf

Mougeon R., Nadasdi, T. et Rehner, K. (2002). État de la recherche sur l'appropriation de la variation par des apprenants avancés du FL2 ou FLE. Acquisition et interaction en langue étrangère, 17, 7-50.

Nardy, A., Chevrot, J.-P. et Barbu, S. (2013). The acquisition of sociolinguistic variation: Looking back and thinking ahead. Linguistics, 51(2), 255-284.

Paquet-Gauthier, M. (2015). Sens influencés de l'anglais en français au Québec: utilisation, perception et intégration (Mémoire de maîtrise inédite). Université Laval, Ville de Québec, QC. Repéré à https://www.researchgate.net/publication/287646599_Sens_influences_de_l'anglais _en_francais_au_Quebec_utilisation_perception_et_integration

Preston, D. R. (2013). The influence of regard on language variation and change. Journal of Pragmatics, 52, 93-104.

von Münchow, P. (2004a). Comparaison de manuels de français et d'allemand langue maternelle : représentations de l'enfant, de l'adulte, de l'apprentissage et de la langue. Marges linguistiques.

von Münchow, P. (2004b). Réflexions sur une linguistique de discours comparative : le cas du journal télévisé en France et en Allemagne. Revue Tranel, 40, 47-70.

von Münchow, P. (2009). Le discours d'adolescents dans les manuels scolaires de lecture en France et en Allemagne. Adolescence, 4(70), 921-930. doi :

10.3917/ado.070.0921

Wachs, S. (2005). Passer les frontières des registres en français : un pas à l'école. Synergies France, 4, 169-177.

Wieczorek, J. A. (1991). Spanish dialects and the foreign language textbook: A sound perspective. Hispania, 74(1), 175-181.

\section{Corpus de manuels}

Archambault, L., Duval, L., Henrichon, L. et Popica, M. (2011). À mots découverts : langue française et communication. Anjou, QC : Éditions CEC.

Augé, H., Cañada Pujols, M.-D., Marthens, C. et Martin, C. (2005a). Tout va bien! 2 : méthode de français. Paris, France : CLE international. 
Augé, H., Cañada Pujols, M.-D., Marthens, C. et Martin, C. (2005b). Tout va bien! 3 : méthode de français. Paris, France : CLE international.

Capelle, G. et Gidon, N. (2002). Reflets 2 : méthode de français. Paris, France : Hachette. Devey, L., Chamberland, J. et Bernard, V. (2010). Transmission 5. Anjou, QC : Éditions CEC.

Devey, L., Chamberland, J. et Pagé, M. (2009). Transmission 4. Anjou, QC : Éditions CEC. Dollez, C. et Pons, S. (2002). Reflets 3 : méthode de français. Paris, France : Hachette. Gaudreault, A. et Quevillon, J. (2004). À propos : $4^{e}$ secondaire. Anjou, QC : Éditions CEC.

Girard, M. et St-Cyr, N. (2004). À propos : $5^{e}$ secondaire. Anjou, QC : Éditions CEC. Mérieux, R., Loiseau, Y. et Bouvier, B. (2005). Connexions : méthode de français (niveau 3). Paris, France : Didier. 